\title{
Serum lactate as predictor of morbidity, mortality and long term survival in patients undergoing cytoreductive surgery and hyperthermic intraperitoneal chemotherapy
}

\author{
John Spiliotis*1, Evgenia Halkia ${ }^{1,2}$, Andrew Zouridis ${ }^{1}$, Dimitra Vassiliadou ${ }^{1}$, Maria Zakka ${ }^{3}$, Nafsika Kalantzi ${ }^{4}$, \\ Archondia Vaxevanidou ${ }^{5}$, Alexandra Pagoulatou ${ }^{4}$, Nikolaos Vaos ${ }^{3}$, Elias Efstathiou ${ }^{1}$, Anastassios Datsis ${ }^{6}$ \\ ${ }^{1}$ Department of Surgical Oncology, Metaxa Cancer Hospital, Piraeus, Greece \\ ${ }^{2}$ Peritoneal Surface Malignancy Unit, IASO General Hospital, Athens, Greece \\ ${ }^{3}$ Intensive Care Unit, Metaxa Cancer Hospital, Piraeus, Greece \\ ${ }^{4}$ Department of Anesthesiology, Metaxa Cancer Hospital, Piraeus, Greece \\ ${ }^{5}$ Department of Anesthesiology, Gennimatas General Hospital, Thessaloniki, Greece \\ ${ }^{6}$ Department of Surgery, General Hospital of Mesolonghi, Greece
}

Received: June 28, 2015

DOI: $10.5430 /$ css.v1n $1 \mathrm{p} 41$
Accepted: August 5, 2015

Online Published: August 14, 2015

\begin{abstract}
Introduction: Serum lactate elevation has been used as a marker of tissue hypoxia in the setting of perioperative monitoring and critical care. It has also been correlated with perioperative morbidity and mortality. Cytoreductive surgery (CRS) \& hyperthermic intraperitoneal chemotherapy (HIPEC) for the treatment of peritoneal carcinomatosis is a major abdominal operation, requiring meticulous perioperative care. The aim of this study is to assess the clinical use of lactate measurements after CRS \& HIPEC and its prognostic value in terms of immediate postoperative morbidity and mortality, as well as long term survival.

Material \& Methods: Of 140 patients diagnosed with peritoneal carcinomatosis who underwent cytoreductive surgery and HIPEC were prospectively studied. Serum lactate was measured by air blood gases analyser intraoperatively (just before the administration of HIPEC) and then daily till the fifth postoperative day. Postoperative complications were recorded and divided into two groups according to Clavien Dindo classification.

Results: Intraoperative lactate measurements have not been associated with postoperative morbidity and mortality. On the other hand, lactate measurements on postoperative days 3 and 4 are of clinical significance. Specifically, an increase of $1 \mathrm{mmol} / \mathrm{L}$ of the average lactate value of days 3 and 4 raises the risk of a minor complication (Grades I to IIIa) by 1.9, the risk of a major complication (Grades IIIb to V) by 10.9 and the risk of mortality by $32.1 \%$.

Conclusions: The average of day 3 and 4 postoperative day lactate level is an independent predictor of morbidity and mortality in patients undergoing CRS and HIPEC.
\end{abstract}

Key Words: Cytoreductive surgery, Hyperthermic intraperitoneal chemotherapy, Lactate, Perioperative management Greece.

*Correspondence: John Spiliotis; Email: jspil@in.gr; Address: 1st Department of Surgical Oncology, Metaxa Cancer Hospital, 185 37, Piraeus, 


\section{INTRODUCTION}

Cytoreductive surgery (CRS) and hyperthermic intraperitoneal chemotherapy (HIPEC) is a novel therapeutic modality in the approach of peritoneal carcinomatosis, a previously considered fatal condition. CRS consists of a series of peritonectomy procedures, as described by Sugarbaker, ${ }^{[1]}$ aiming to remove all macroscopically visible disease. After cytoreduction, the peritoneal cavity is perfused with a chemotherapeutic agent at an increased temperature (HIPEC). Therefore, CRS \& HIPEC can be viewed as a major abdominal operation, requiring meticulous monitoring over the intraoperative and postoperative period, in order to achieve optimal resuscitation.

Serum lactate has proven to be a useful marker of adequate resuscitation, its elevation being indicative of tissue hypoxia, as well as predictive of morbidity and mortality. ${ }^{[2,3]}$

The aim of this study is to assess the clinical use of lactate measurements after CRS \& HIPEC and its prognostic value in terms of immediate postoperative morbidity and mortality, as well as long term survival.

\section{MATERIAL AND METHODS}

Over a period of six years (2008-2013), 140 patients diagnosed with peritoneal carcinomatosis who underwent cytoreductive surgery and HIPEC were prospectively studied. The primary endpoints of the study were perioperative morbidity and mortality in association with serum lactate levels as well as overall survival. Peritoneal carcinomatosis was either primary (mesothelioma) or metastatic (pseudomyxoma, colorectal, ovarian, gastric). The study was approved by our institution's Ethical Committee and all patients agreed to be included in the study, signing an informed consent form.

\subsection{Data collection}

Serum lactate was measured by air blood gases analyser intraoperatively (just before the administration of HIPEC) and then daily till the fifth postoperative day. Postoperative complications were recorded and divided into two groups according to Clavien Dindo classification. ${ }^{[4]}$ Fatal complications (Grade V) and complications demanding ICU management (Grade IV) or surgical intervention under general aneasthesia (Grade IIIb) were listed as major. All the other complications (Grade I - IIIa) were characterized minor. Peritoneal carcinomatosis was estimated using the Peritoneal Cancer Index (PCI) which ranges from 1 to 39. ${ }^{[5]}$ Cytoreduction result after CRS was assessed using the Completeness of Cytoreduction Score (CCS) which ranges from 0 to $3 .^{[6]}$ Demographic characteristics were also collected (age, gender). Patient follow up was performed every three months for the first year and every six months thereafter.

\subsection{Cytoreductive surgery and HIPEC procedure}

Anaesthetic management was performed according to hospital protocols, adjusted to the special demands of CRS and HIPEC,$^{[7-9]}$ and patients' individual features. All the patients were operated on by the same surgical team. After midline laparotomy and PCI assessment, ${ }^{[5]}$ extensive cytoreduction was performed, using the Sugarbaker technique (peritonectomies). ${ }^{[1,10]}$ After the evaluation of cytoreduction, using CC score ${ }^{[6]}$ HIPEC was delivered for 60 to 90 minutes using either the closed or the open (coliseum) technique. ${ }^{[1]}$ The used chemotherapeutic agents depended on the tumor histological features. The temperature of the infused solution was $41^{\circ} \mathrm{C}-42^{\circ} \mathrm{C}$. Postoperatively $20 \%$ of the patients were admitted to the ICU for at least one day.

\subsection{Statistical analysis}

To investigate whether serum lactate levels are related to postoperative complications we used the Mann Whitney test. Moreover, to account for potential confounders we used logistic regression. Specifically, in all cases we adjusted for potential effects of PCI and CCS based on prior knowledge ${ }^{[12]}$ and independent of the estimated $p$-value; as for the rest of the confounders, we included them in the final model only if they had statistically significant results.

Survival was evaluated using Kaplan - Meier analysis. To quantify the effect of lactate in survival time while adjusting for potential confounders, we used Cox proportional hazards model.

A 2 -sided $p$-value $<.05$ was considered statistically significant. All analysis was performed in IBM SPSS v.22.

\section{RESUlts}

One hundred and forty patients underwent CRS and HIPEC were investigated. Details for the patients demographics, the origin of peritoneal carcinomatosis, the procedures performed and the outcomes are given in Table 1.

\subsection{Lactate and morbidity}

Forty three patients $(30.7 \%)$ were reported with minor complications. Although the median intraoperative lactate levels between patients with $(2.9 \mathrm{mmol} / \mathrm{L})$ and without (1.9 mmol/L) minor complications had a statistically significant difference ( $p=.006)$, after adjustment for PCI and CCS, intraoperative lactate levels did not differ significantly ( $p=$ .160). On the other hand, average day 3 and 4 lactate levels had a statistically significant difference between patients with and without minor complications (see Figure 1) and for each one unit increase in average day 3 and 4 lactate levels, the probability of having a minor event raises by 1.9 times $(95 \%$ C.I. $=[1.40,2.57])$, after adjusting for PCI and CCS. 
Table 1. Descriptive statistics $(n=140)$

\begin{tabular}{|c|c|c|}
\hline & $\mathbf{n}$ & $\%$ \\
\hline \multicolumn{3}{|l|}{ Gender } \\
\hline Male & 100 & 71.4 \\
\hline Female & 40 & 29.6 \\
\hline \multicolumn{3}{|l|}{ Age } \\
\hline$<36$ & 13 & 9.3 \\
\hline $36-50$ & 51 & 36.4 \\
\hline $51-65$ & 46 & 32.9 \\
\hline$>65$ & 30 & 21.4 \\
\hline \multicolumn{3}{|c|}{ Origin of peritoneal carcinomatosis } \\
\hline Colorectal & 48 & 34.3 \\
\hline Ovarian & 31 & 22.1 \\
\hline Gastric & 21 & 15 \\
\hline Pseudomyxoma & 19 & 13.6 \\
\hline Mesothelioma & 17 & 12.1 \\
\hline Sarcoma & 4 & 2.9 \\
\hline \multicolumn{3}{|c|}{ Peritoneal Carcinomatosis Index } \\
\hline $\mathrm{PCI}<13$ & 36 & 25.7 \\
\hline $13 \leq \mathrm{PCI}<26$ & 77 & 55.0 \\
\hline $\mathrm{PCI} \geq 26$ & 27 & 19.3 \\
\hline \multicolumn{3}{|l|}{ Operations Performed } \\
\hline splenectomy & 49 & 35 \\
\hline cholecystectomy & 130 & 92.9 \\
\hline omentectomy & 132 & 94.3 \\
\hline hysterectomy & 27 & 19.3 \\
\hline gastrectomy & 19 & 13.5 \\
\hline Douglas resection & 24 & 17.1 \\
\hline small bowel resection & 127 & 90.7 \\
\hline partial colectomy & 39 & 27.8 \\
\hline complete colectomy & 8 & 5.7 \\
\hline \multicolumn{3}{|c|}{ Completeness of Cytoreduction } \\
\hline $\mathrm{CC}-0$ & 98 & 70 \\
\hline $\mathrm{CC}-1$ & 33 & 23.5 \\
\hline $\mathrm{CC}-2$ & 9 & 6.5 \\
\hline \multicolumn{3}{|l|}{ Complications } \\
\hline Minor & 43 & 30.7 \\
\hline Major & 39 & 27.8 \\
\hline \multicolumn{3}{|l|}{ Survival } \\
\hline 1 year & 127 & 91 \\
\hline 2 years & 96 & 69 \\
\hline 3 years & 85 & 61 \\
\hline
\end{tabular}

In 39 patients (27.8\%) major complications were observed. The median intraoperative lactate levels between patients with and without major complications differ significantly $(p=.001)$. After adjustment for age and PCI intraoperative lactate levels were not statistical significant $(p=.143)$. As regards the average day 3 and 4 lactate levels, a statistically significant difference has been proven between patients Published by Sciedu Press with and without major complications (see Figure 2) and for each one unit increase, the probability of having a major event raises by 10.9 times (95\% C.I. $=[4.37,26.90])$, after adjusting for PCI.

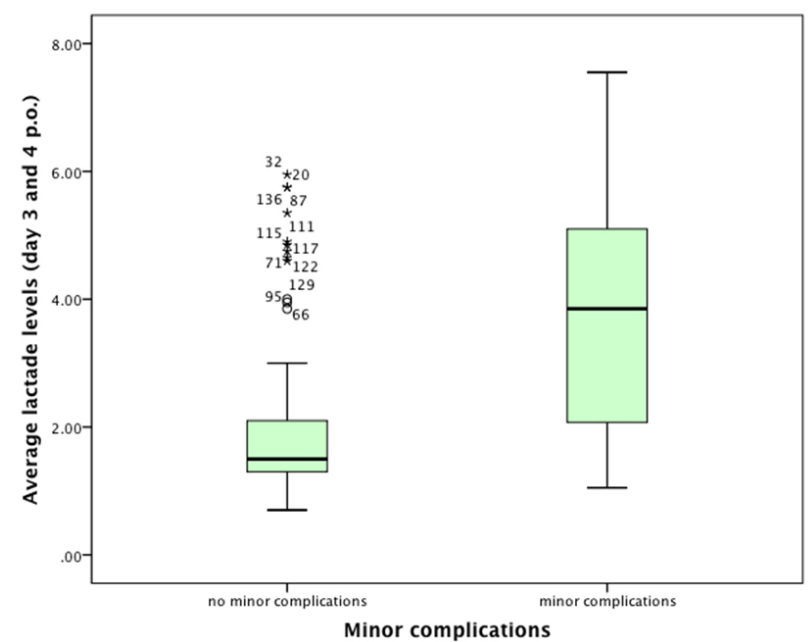

Figure 1. Minor complications

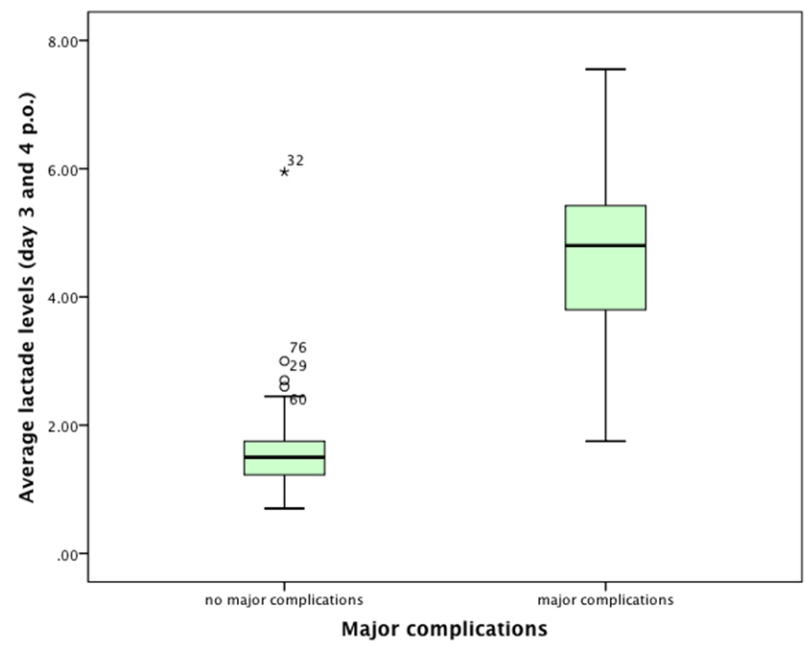

Figure 2. Major complications

\subsection{Lactate and survival}

Kaplan Meier analysis shows that the survival times for each lactate level category intraoperatively were not equal $(p<.05)$. However, when we used Cox proportional hazards model and adjusted for age, PCI and CCS, the results of intraoperative lactate levels were not statistically significant $(p=.233)$.

3rd and 4th postoperative day average lactate groups had different survival according to Kaplan Meier analysis $(p<.05)$ (see Figure 3). Patients' liver and renal function tests were within normal limits. The cutoff values we used for grouping 
the patients were $1.65 \mathrm{mmol} / \mathrm{L}(0.35$ above our analyser's upper limit of normal) and $2.65 \mathrm{mmol} / \mathrm{L}$ (since in literature a value of 2.0-2.5 is considered elevated). Cox regression in addition shows that there is a $32.1 \%$ increase in the expected hazard of mortality associated with an one unit increase in the average of day 3 and 4 lactate levels, adjusting for age, PCI and CCS $(p=.004)$.

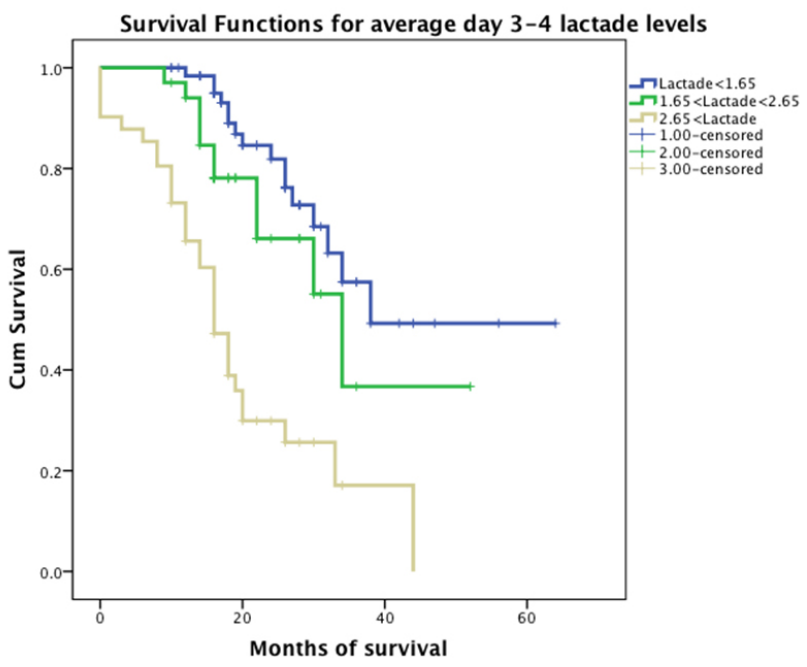

Figure 3. Survival analysis

The above data conclude that the average of day 3 and 4 postoperative day lactate level is an independent predictor of morbidity and mortality in patients undergoing CRS and HIPEC.

\section{Discussion}

\subsection{Lactate physiology and hyperlactaemia}

Lactate is produced by most tissues in the human body, its main production occurring in muscle tissue. Under normal conditions, it is rapidly cleared by the liver, and additionally by the kidneys. In aerobic conditions, pyruvate is produced via glycolysis and then enters the Krebs cycle, largely bypassing the production of lactate. Under anaerobic conditions, lactate is an end product of glycolysis and feeds into the Cori cycle as a substrate for gluconeogenesis. ${ }^{[13]}$ It is increased in conditions of tissue hypoperfusion or stress leading to increased glycolysis. Although lactate may be elevated in several conditions, it has been effectively used as a measure of tissue hypoxia. ${ }^{[14-16]}$

Normal serum lactate values are $0.3-1.3 \mathrm{mmol} / \mathrm{L}$, with several studies using 2.0-2.5 mmol/L as cutoff values for elevated lactate, while in most studies "high" lactate is considered when it measures more than $4.0 \mathrm{mmol} / \mathrm{L}$.

The aetiology of hyperlactaemia is:
- increased lactate production: increased glycolysis, enzyme defects

- decreased hepatic lactate clearance: impaired oxidative metabolism (impaired liver blood flow), impaired glyconeogenesis (oral hypoglycaemic drugs, Hartmann's solution), chronic liver disease

- sepsis

- decreased extra-hepatic metabolism

- reduced renal excretion ${ }^{[17]}$

\subsection{Intraoperative \& perioperative management of CRS \& HIPEC patients}

Patient status should be optimized preoperatively, as peritoneal carcinomatosis patients are often suboptimal surgical candidates, with factors such as older age, comorbidities and cachexia complicating their perioperative course.

Cytoreductive surgery is a major abdominal operation, consisting of peritonectomies and extensive visceral resections, causing significant fluid, blood and protein losses, increased intra-abdominal pressure, systemic hyperthermia and increased metabolic rate therefore significant pathophysiological disturbances. ${ }^{[8]}$

HIPEC involves the rinsing of the abdominal cavity with a heated chemotherapy solution. Most regimens suggest the administration of the chemoperfusate for 60 to 120 minutes, at $42^{\circ} \mathrm{C}$. The chemotherapeutic agent used depends on the site of the initial neoplasia, the most commonly used being mitomycin-c, oxaliplatin, irinotecan and cisplatin.

A retrospective analysis of 78 patients undergoing cytoreductive surgery \& HIPEC demonstrated a large intraoperative fluid turnover, increased airway pressure and central venous pressure (due to the increased intraabdominal pressure with the closed technique), while increased body temperature resulted in a mild metabolic acidosis. ${ }^{[9]}$

According to the findings of another prospective study of 60 patients, haemodynamic disturbances occurred during HIPEC administration, characterized by an increase in heart rate and cardiac output and a decreased systemic vascular resistance on account of increased body temperature and decreased effective circulating volume. Urinary output showed a decreasing tendency over time. ${ }^{[18]}$

HIPEC with oxaliplatin has been associated with severe hyponatraemia, hyperglycaemia and hyperlactaemia, not observed with cisplatin or mitomycin C. ${ }^{[19,20]}$ This was attributed to the use of dextrose $5 \%$ as a carrier for oxaliplatin and to major loss of sodium into the chemoperfusate. ${ }^{[19]}$ Moreover, higher perfusion temperature was related to more pronounced changes in serum glucose, sodium and lactate 
levels. ${ }^{[21]}$

Postoperative ICU admission is often considered protocol after cytoreductive surgery \& HIPEC, mainly due to the need for haemodynamic surveillance and stabilization after this major operation. However, it was recently reported that there was no difference in the rate and degree of complications observed in patients who were admitted in the ICU, noting that ICU admission should not be standardized, but based on individual patient characteristics. ${ }^{[22]}$

Intraoperative parameters may be associated with postoperative outcome, in terms of morbidity and mortality, as demonstrated by a previous study conducted by our team. ${ }^{[23]}$

\subsection{Lactate as a marker of perioperative and long - term outcome}

The findings of this study suggest that while intraoperative serum lactate levels do not indicate the postoperative course, it is quite on the contrary with lactate levels on postoperative days 3 and 4 . Specifically, an increase of $1 \mathrm{mmol} / \mathrm{L}$ of the average lactate value of days 3 and 4 raises the risk of a minor complication (Grades I to IIIa) by 1.9, the risk of a major complication (Grades IIIb to V) by 10.9 and the risk of mortality by $32.1 \%$.

Several studies have evaluated the clinical use of lactate measurement in postoperative patients, not only in abdominal surgery, ${ }^{[2,24,25]}$ but also in cardiac surgery in adults ${ }^{[3]}$ and children. ${ }^{[26]}$ Ours is the first study to assess the efficacy of lactate as a marker of morbidity and mortality in the postoperative course of patients undergoing cytoreductive surgery and HIPEC.
Lactate has been used as a measure of tissue hypoperfusion, with lactate clearance being used to guide resuscitation. ${ }^{[14-16]}$ Therefore, not only increased lactate levels, but also the time to their normalization, have been associated with postoperative mortality. ${ }^{[16]}$

In a study of 137 surgical intensive care unit patients, elevated lactate values initially and 24 hours later significantly correlated with mortality, indicating lactate as an efficient predictive marker. Moreover, lactate clearance proved to be of great importance, as mortality raised with persistently elevated lactate levels, reaching $67 \%$ if lactate failed to normalize. ${ }^{[2]}$

In a prospective study of 88 patients having undergone major elective abdominal surgery and presented a minor or major complication, it was found that serum lactate levels were significantly increased compared to patients with an uneventful postoperative course. Also the accuracy of lactate to predict morbidity increased with time, and the cut off value was $1.46 \mathrm{mmol} / \mathrm{L}^{[25]}$

Another useful marker to predict reintervention after abdominal surgery is peritoneal lactate, as well as the peritoneal serum lactate ratio. ${ }^{[2]}$

\section{Conclusion}

In conclusion, serum lactate can be effectively used as an indicator of the postoperative course in patients who have undergone cytoreductive surgery \& HIPEC. Intraoperative measurements did not correlate significantly with either morbidity or mortality, while an elevated average lactate value of postoperative days 3 and 4 significantly increased the risk of postoperative complications and mortality.

\section{REFERENCES}

[1] Sugarbaker PH. Peritonectomy procedures. Cancer treatment and research. 2007; 134: 247-64. http://dx.doi.org/10.1007/978 -0-387-48993-3_15

[2] Husain FA, Martin MJ, Mullenix PS, et al. Serum lactate and base deficit as predictors of mortality and morbidity. American journal of surgery. 2003; 185(5): 485-91. http://dx.doi.org/10.1016/S 0002-9610(03) 00044-8

[3] Toraman F, Evrenkaya S, Yuce M, et al. Lactic acidosis after cardiac surgery is associated with adverse outcome. The heart surgery forum. 2004; 7(2): E155-9. PMid: 15138095. http://dx.doi.org/10. 1532/HSF98. 20041002

[4] Dindo D, Demartines N, Clavien PA. Classification of surgical complications: a new proposal with evaluation in a cohort of 6336 patients and results of a survey. Annals of surgery. 2004; 240(2): 205-13. PMid: 15273542. http://dx.doi.org/10.1097/01.sla.0000 133083.54934.ae

Published by Sciedu Press
[5] Jacquet P, Sugarbaker PH. Clinical research methodologies in diagnosis and staging of patients with peritoneal carcinomatosis. In: Sugarbaker PH, editor. Peritoneal Carcinomatosis: Principles of Management: Springer Science \& Business Media; 1996. http: //dx.doi.org/10.1007/978-1-4613-1247-5_23

[6] Sugarbaker PH, Chang D. Results of treatment of 385 patients with peritoneal surface spread of appendiceal malignancy. Annals of surgical oncology. 1999; 6(8): 727-31. http://dx.doi.org/10.10 07/s10434-999-0727-7

[7] Kajdi ME, Beck-Schimmer B, Held U, et al. Anaesthesia in patients undergoing cytoreductive surgery with hyperthermic intraperitoneal chemotherapy: retrospective analysis of a single centre three-year experience. World journal of surgical oncology. 2014; 12: 136. PMid: 24886171. http://dx.doi.org/10.1186/1477-7819-12-136

[8] Raspe C, Piso P, Wiesenack C, et al. Anesthetic management in patients undergoing hyperthermic chemotherapy. Current opinion in anaesthesiology. 2012; 25(3): 348-55. PMid: 22517311. http: 
//dx.doi.org/10.1097/ACO.0b013e32835347b2

[9] Schmidt C, Creutzenberg M, Piso P, et al. Peri-operative anaesthetic management of cytoreductive surgery with hyperthermic intraperitoneal chemotherapy. Anaesthesia. 2008; 63(4): 389-95. PMid: 18336490. http://dx.doi.org/10.1111/j.1365-2044. 2007 $.05380 . x$

[10] Halkia E, Efstathiou E, Spiliotis J, et al. Management of diaphragmatic peritoneal carcinomatosis: surgical anatomy guidelines and results. Journal of BUON : official journal of the Balkan Union of Oncology. 2014; 19(1): 29-33.

[11] Glehen O, Cotte E, Kusamura S, et al. Hyperthermic intraperitoneal chemotherapy: nomenclature and modalities of perfusion. Journal of surgical oncology. 2008; 98(4): 242-6. PMid: 18726885. http://dx.doi.org/10.1002/jso.21061

[12] Spiliotis JD, Halkia E, Boumis VA, et al. Cytoreductive surgery and HIPEC for peritoneal carcinomatosis in the elderly. International journal of surgical oncology. 2014; 2014: 987475. PMid: 24809147. http://dx.doi.org/10.1155/2014/987475

[13] Andersen LW, Mackenhauer J, Roberts JC, et al. Etiology and therapeutic approach to elevated lactate levels. Mayo Clinic proceedings. 2013; 88(10): 1127-40. PMid: 24079682. http://dx.doi.org/1 $0.1016 /$ j.mayocp. 2013.06.012

[14] Jeng JC, Jablonski K, Bridgeman A, et al. Serum lactate, not base deficit, rapidly predicts survival after major burns. Burns : journal of the International Society for Burn Injuries. 2002; 28(2): 161-6. http://dx.doi.org/10.1016/S0305-4179(01) 00098-5

[15] McNelis J, Marini CP, Jurkiewicz A, et al. Prolonged lactate clearance is associated with increased mortality in the surgical intensive care unit. American journal of surgery. 2001; 182(5): 481-5. http://dx.doi .org/10.1016/S0002-9610(01)00755-3

[16] Weil MH, Afifi AA. Experimental and clinical studies on lactate and pyruvate as indicators of the severity of acute circulatory failure (shock). Circulation. 1970; 41(6): 989-1001. http://dx.doi.org /10.1161/01.CIR.41.6.989

[17] Phypers B, Pierce JT. Lactate physiology in health and disease. Continuing Education in Anaesthesia, Critical Care \& Pain. 2006; 6(3): 128-32. http://dx.doi.org/10.1093/bjaceaccp/mk1018

[18] Rankovic VI, Masirevic VP, Pavlov MJ, et al. Hemodynamic and cardiovascular problems during modified hyperthermic intraperitoneal perioperative chemotherapy. Hepato-gastroenterology. 2007; 54(74): 364-6. PMid: 17523275.
[19] De Somer F, Ceelen W, Delanghe J, et al. Severe hyponatremia, hyperglycemia, and hyperlactatemia are associated with intraoperative hyperthermic intraperitoneal chemoperfusion with oxaliplatin. Peritoneal dialysis international : journal of the International Society for Peritoneal Dialysis. 2008; 28(1): 61-6.

[20] Raft J, Parisot M, Marchal F, et al. [Impact of the hyperthermic intraperitoneal chemotherapy on the fluid-electrolytes changes and on the acid-base balance]. Annales francaises d'anesthesie et de reanimation. 2010; 29(10): 676-81. PMid: 20797835. http: //dx.doi.org/10.1016/j.annfar.2010.06.021

[21] Ceelen W, De Somer F, Van Nieuwenhove Y, et al. Effect of perfusion temperature on glucose and electrolyte transport during hyperthermic intraperitoneal chemoperfusion (HIPEC) with oxaliplatin. European journal of surgical oncology : the journal of the European Society of Surgical Oncology and the British Association of Surgical Oncology. 2013; 39(7): 754-9. PMid: 22878060. http://dx.doi.org/10.1016/j.ejso.2012.07.120

[22] Lopez-Basave HN, Morales-Vasquez F, Mendez-Herrera C, et al. Intensive care unit admission after cytoreductive surgery and hyperthermic intraperitoneal chemotherapy. Is it necessary? Journal of oncology. 2014; 2014: 307317. http://dx.doi.org/10.1155/2 $014 / 307317$

[23] Spiliotis J, Vaxevanidou A, Datsis A, et al. Peritoneal carcinomatosis: intra-operative and post-operative assessment of patients undergoing cytoreduction and HIPEC. Hepato-gastroenterology. 2010; 57(102103): 1052-9. PMid: 21410030.

[24] Bini R, Ferrari G, Apra F, et al. Peritoneal lactate as a potential biomarker for predicting the need for reintervention after abdominal surgery. The journal of trauma and acute care surgery. 2014; 77(2): 376-80. PMid: 25058267. http://dx.doi.org/10.1097/TA. 00 00000000000302

[25] Li S, Peng K, Liu F, et al. Changes in blood lactate levels after major elective abdominal surgery and the association with outcomes: a prospective observational study. The Journal of surgical research. 2013; 184(2): 1059-69. PMid: 23721936. http: //dx.doi.org/10.1016/j.jss.2013.04.056

[26] Hatherill M, Sajjanhar T, Tibby SM, et al. Serum lactate as a predictor of mortality after paediatric cardiac surgery. Archives of disease in childhood. 1997; 77(3): 235-8. PMid: 9370903. http://dx.doi.org/10.1136/adc.77.3.235 